\begin{tabular}{|c|c|c|}
\hline & Int.J.Curr.Microbiol.App.Sci (2016) 5(10): 842-848 & \multirow{2}{*}{ 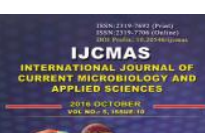 } \\
\hline & \multirow{4}{*}{$\begin{array}{l}\text { International Journal of Current Microbiology and Applied Sciences } \\
\text { ISSN: 2319-7706 Volume } 5 \text { Number } 10 \text { (2016) pp. 842-848 } \\
\text { Journal homepage: http://www.ijcmas.com }\end{array}$} & \\
\hline & & \\
\hline EXCELLENT & & \\
\hline PUBLISHERS & & wwwijijemas com \\
\hline
\end{tabular}

Original Research Article

http://dx.doi.org/10.20546/ijcmas.2016.510.092

\title{
Study of Acinetobacter Isolates from Clinical Specimens in Tertiary Care Hospital and their Antimicrobial Susceptibility Pattern
}

\author{
Harekrishna Nath* and Dipa Barkataki
}

\author{
Department of Microbiology, Gauhati Medical College and Hospital, \\ Guwahati, Kamrup Assam, India \\ *Corresponding author
}

\begin{abstract}
A B S T R A C T
Keywords

Acinetobacter, gram negative bacilli,

ICU infection, MBL producer.

Article Info

Accepted:

25 September 2016

Available Online:

10 October 2016

Acinetobacter spp play a significant role in the colonization and infection of patients admitted to hospitals. Acinetobacter is a Gram Negative Coccobacillus. Outbreaks of infection caused by strains of A. baumannii resistant to multiple antibiotic classes, including carbapenems, are a serious concern in many specialized hospital units, including intensive care units (ICUs). Given the diversity of resistance mechanisms in A. baumannii, definitive therapy should be based on the results of antimicrobial susceptibility testing. Keeping these things mind the present study was undertaken to study the prevalence of Acinetobacter spp in clinical samples and the antimicrobial susceptibility pattern's A total of 888 no's clinical specimens Urine, Respiratory samples, Blood wound swab, Body fluids collected from admitted patients in wards and ICU department of GMCH for a period of one year from August 2014--July 2015. Acinetobacter isolation was done by conventional methods of culture. Species identification was done by KBO14 and by Vitek2 compact. Antimicrobial susceptibility of isolated Acinetobacter spp were tested by modified Kirby-Bauer disc diffusion method as per the recommendation of Clinical and Laboratory Standard Institute (CLSI) and VITEK 2 compact AST 281 as per guideline. Out of 888 clinical samples (31) Acinetobacter spp were isolated and prevalence was $3.49 \%$. The maximum isolation rate was seen from respiratory specimens $(45.16 \%)$ followed by urine (19.36\%), wound swabs (16.13\%), blood (12.90\%) and body fluid (6.45\%). Acinetobacter baumannii was the predominant species $(90.32 \%)$ isolated, followed by Acinetobacter lwoffii (6.45\%) and Acinetobacter hemolyticus (3.23\%). Acinetobacter species isolated from patients in ICU was $80.65 \%(25)$. Acinetobacter baumannii were sensitive to Polymixin B (100\%) followed by colistin (92.85\%).The highest resistance was seen with Piperacillin tazobactum (71.43).Carbapenem resistance found in $42.85 \%$ A.baumannii. Acinetobacter spp. 38.7\% (12) of isolates were MBL producers and all MBL producers were isolated from ICU.
\end{abstract}

\section{Introduction}

Acinetobacter spp play a significant role in the colonization and infection of patients admitted to hospitals (Bergogne-Berezin et al., 1996). Acinetobacter is a Gram Negative Coccobacillus (Munoz-Price et al., 2008; Schreckenberger et al., 2007) that during the past three decades has emerged from an organisms of questionable pathogenicity to an infectious agent of importance worldwide (Fournier et al., 2006; Dima et al., 2007). Acinetobacter infection have long being clinically prominent in tropical countries, have been recurrent problems during war and natural disasters, and have 
recently caused multihospital outbreak in temperate climates (Lolans et al., 2006).

It is an opportunistic pathogen found to be associated with a wide spectrum of infection including nosocomial pneumonia, meningitis, endocarditis skin and soft tissue infections, urinary tract infection, conjunctivitis, burn wound infections and bacteramia. Acinetobacter baumannii is the commonest isolates from Gram- negative sepsis in immune compromised patients, posing risk for high mortality (Patwardhan et al., 2008).

It typically colonizes skin, the respiratory, urinary, gastrointestinal tract wounds and indwelling plastic devices of the hospitalized patients can cause infections in burn, trauma, mechanically ventilated and immunocompromised patients. It shows a special predilection for the ICU (Namita Jaggi et al., 2012) and more than two third of Acinetobacter infections are due to Acinetobacter baumannii.

Acinetobacter spp is easily isolated in standard cultures but is relatively nonreactive in many biochemical tests commonly used to differentiate among gram-negative bacilli. This can delay isolate identification by a day. A. baumannii, A. calcoaceticus, and A. lwoffii are the Acinetobacter species most frequently reported in the clinical literature. Because it is difficult to differentiate among Acinetobacter species on the basis of phenotypic characteristics, the term $A$. calcoaceticus-A. baumannii complex is sometimes used. Rational use of antimicrobial agents is critically important to prevent Acinetobacter infections as well as to avoid poor outcomes. Carbapenems have long been thought of as the agents of choice for serious A. baumannii infections. However, the clinical utility of carbapenems is increasingly jeopardized by the production of carbapenemases. Outbreaks of infection caused by strains of $A$. baumannii resistant to multiple antibiotic classes, including carbapenems, are a serious concern in many specialized hospital units, including intensive care units (ICUs). The foremost implication of infection with carbapenem-resistant $A$. baumannii is the need to use "last-line" antibiotics such as colistin, polymyxin B, or Tigecycline (David et al., 2012).

Selection of empirical antibiotic therapy when A. baumannii is suspected is challenging and must rely on knowledge of local epidemiology. The interval from onset of infection to initiation of effective empirical therapy clearly influences outcome. Given the diversity of resistance mechanisms in $A$. baumannii, definitive therapy should be based on the results of antimicrobial susceptibility testing. Keeping these things mind the present study was undertaken with following aims and objectives.

The main objective of this study includes, to study the prevalence of Acinetobacter spp in clinical samples of GMCH. And also to study the antimicrobial susceptibility pattern of the isolated Acinetobacter spp.

\section{Materials and Methods}

A total of 888 no's clinical specimens Urine, Respiratory samples (Sputum, endotracheal aspirate and bronchoalveolar lavage), Blood /catheter tips, Pus/ wound swab, Body fluids(Pleural fluid, ascetic fluid and cerebrospinal fluid etc) collected from different medical wards, surgical wards and ICU department of GMCH for a period of one year from August 2014--July 2015. The study was commenced with ethical approval and clearance certificate from the IEC, $\mathrm{GMCH}$. 


\section{Inclusion criteria}

All gram negative coccobacilli, strict aerobes, nonfermenter, catalase positive, oxidase negative isolates (from Hospitalized patients Patients on a ventilator, Prolonged hospital stay, Open wounds, Person with invasive devices like urinary catheters, ICU patients) were considered

\section{Exclusion criteria}

OPD patients and Organism other than Acinetobacter spp isolated from clinical specimens was excluded from the study. Acinetobacter isolation was done by conventional methods of culture. Species identification was done by KBO14 and by Vitek2 compact. Antimicrobial susceptibility of isolated Acinetobacter spp were tested by modified Kirby-Bauer disc diffusion method as per the recommendation of Clinical and Laboratory Standard Institute (CLSI)and VITEK 2 compact AST 281) as per guideline. Acinetobacter baumannii ATCC19606 and Acinetobacter lwoffii ATCC 15309 were used as quality control strains. VITEK 2 compact -identification and sensitivity testing confirmation done by (GN card and AST 281) as per guideline.

\section{Results \& Discussion}

Out of 888 clinical samples Acinetobacter spp were isolated and prevalence was $3.49 \%$.Out of the total of 31 Acinetobacter isolates, the maximum isolation rate was seen from respiratory specimens $(45.16 \%)$ followed by urine (19.36\%), wound swabs (16.13\%), blood (12.90\%) and bodyfluid (6.45\%). Acinetobacter baumannii was the predominant species $(90.32 \%)$ isolated, followed by Acinetobacter lwoffii (6.45\%) and Acinetobacter hemolyticus (3.23\%) Acinetobacter baumannii was the most frequently isolated species from the clinical specimens like respiratory, urine, wound swabs, blood and body fluids. Acinetobacter lwoffii was the next common species isolated from clinical specimens. The single isolate A.hemolyticus was isolated from specimens of blood. Acinetobacter species isolated from patients in ICU was $80.65 \%(25)$ and other Non ICU hospitalized patients showed lower isolation rates $19.35 \%$.Acinetobacter baumannii was $96 \%$ ( 24) followed by A. lwoffii 4\%(1) in ICU. Interestingly, lower isolation rates $19.35 \%$ (6) in Non ICU patients of which Acinetobacter baumannii was predominant $66.66 \%$ (4) followed by A.lwoffii (16.67\%) and A.hemolyticus(16.67\%).The result of sensitivity test Acinetobacter baumannii were sensitive to Polymixin B (100\%) followed by colistin $(92.85 \%)$. Tigecycline and Minocycline were the next most effective antibiotics $(82.14 \%)$, followed by Amikacin (71.43\%) and cefoperazone sulbactum(64.85\%).Highest resistance seen with Piperacillin tazobactum (71.43\%) followed by Aztreonam (60\%), doxycycline $(57.14 \%)$.

Carbapenem resistance found in $42.85 \%$ A.baumannii. The antimicrobial susceptibility tests of the A.baumannii showed more resistant pattern to antimicrobial agents than A.lwoffii and A.hemolyticus. The single isolate A.hemolyticus found (100\%) sensitive to all of the antibiotics plated. Acinetobacter spp. $38.7 \%$ (12) of isolates were MBL producer and all MBL producers were isolated from ICU.

In the present study, the highest number of isolates $32.26 \%$ (10) in age group 21-40 years and $51.61 \%(16)$ Acinetobacter spp isolated in male similar to the study done by Sivaranjani V et al., (2013).

Maximum isolation rates were seen from Respiratory samples $45.16 \%$. similarly 
Namita Jaggi et al., (2015) reported maximum isolates from respiratory secretions (57.4\%) High isolation of Acinetobacters in respiratory samples is perhaps due to its ability to survive in moist surfaces which helps in the transmission and propagation of this organism in the hospital setting.

In the study, the isolation rate of Acinetobacter from urine was (19.36\%). similar observation was made by Sivaranjani et al., (2013).

In the present study, out of 31 Acinetobacter spp, the predominant species was A. baumannii(90.32\%) followed by A.lwoffii $(6.45 \%)$ and A.hemolyticus (3.23\%). similarly, Rahul Kamble et al., (2012) and Sinha et al., reported Acinetobacter baumannii as the predominant species. Predominance A.calcoaceticus baumannii complex is perhaps its non fastidious natures and higher prevalence in hospital environment. Acinetobacter spp. have emerged as particularly important organisms in intensive care units (ICUs), and this is probably related, at least in part, to the increasingly invasive diagnostic and therapeutic procedures used in hospital ICUs in recent years.

Table.1 Distribution of species in ICU and Indoor

\begin{tabular}{|l|l|l|l|l|}
\hline Patient & No(\%) of & \multicolumn{3}{|l|}{ Distribution of species(\%) } \\
\cline { 3 - 5 } & Acinetobacter isolates & Acb clx & A.lwoffii & A.hemolyticus \\
\cline { 3 - 5 } & $6(19.35)$ & $4(66.66)$ & $1(16.67)$ & $1(16.67)$ \\
\hline Indoor & $25(80.65)$ & $24(96)$ & $1(4)$ & 0 \\
\hline TCU & $31(100)$ & $28(90.32)$ & $2(6.45)$ & $1(3.23)$ \\
\hline
\end{tabular}

Fig.1 \& Fig 2

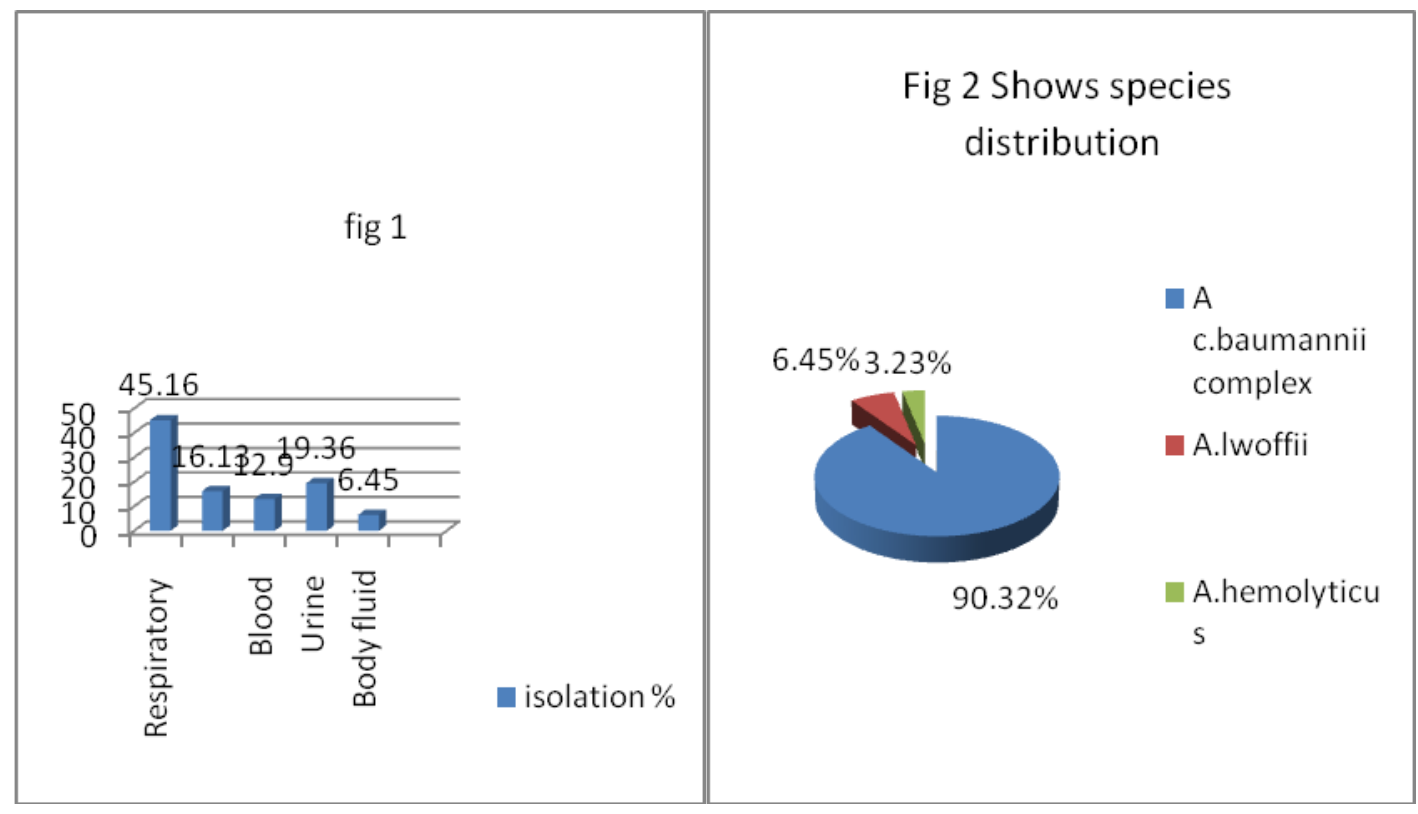




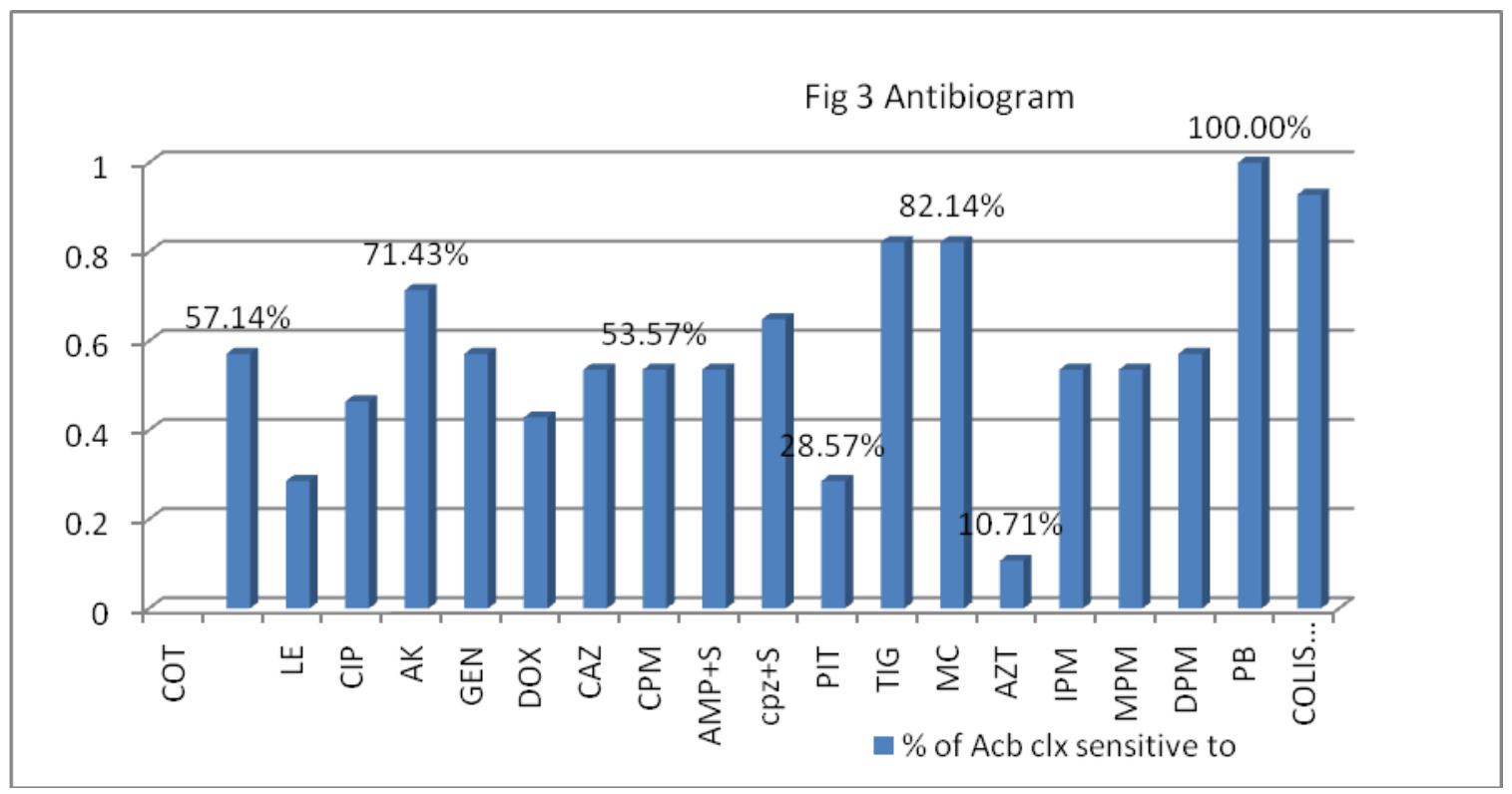

In the present study, Acinetobacter species isolated from patients in ICU most frequently $(80.65 \%)$ and Similarly by Sharma et al., (2015) found Acinetobacter isolates from ICU $71.5 \%$ cases. Muntasir Omer et al., (2014) isolated A. Baumannii from ICU patients $(72 \%)$.

It is observed in our study A.calcoaceticus baumannii complex were sensitive to Polymixin B (100\%).Similar observation was seen in study done by Biglari et al., (2013) and Patwardhan et al., (2003) reported Acinetobacter spp. were $100 \%$ sensitive to Polymixin B.

In the present study Acinetobacter sp showed susceptibility to colistin (93.55\%). Study conducted by Jaggi et al., reported susceptibility of colistin $98.8 \%$. But Dash et al., (July 2010) reported susceptibility of colistin $100 \%$.

In our study resistance was seen with Piperacillin tazobactum (71.43\%).similarly, Chakraborty et al., (2011) reported resistance to Piperacillin/ tazobactam, $(82.9 \%)$. This can be explained by great diversity of chromosomal and plasmidmediated enzymes.
A.lwoffii(2) and (1) A.hemolyticus were sensitive to polymixin $\mathrm{B}$, Tigecycline and Minocycline (100\%) similar with Prasantha et al., (2004).

In conclusion, after analyzing it was concluded that Acinetobacter isolates constitute $3.49 \%$ with a highest isolation from ICUS $(80.65 \%)$. Hospital strains of Acinetobacter showed resistant to commonly used antibiotics. The increasing antibiotic resistant trend reflects the extensive usage of antibiotics in hospitals and its propensity to acquire antibiotic resistance.

In our study Acinetobacter spp were only $100 \%$ sensitive to Polymixin B followed by colistin 92.85\%. Highest resistance seen with Piperacillin tazobactum $71.43 \%$ So, it is essential to implement infection control measures and antibiotic control strategy in hospitals to prevent multidrug resistance by regular analysis of antibiogram of Acinetobacter species. So that an effective empirical drug regime can be tailored to prevent drug resistance and thus the morbidity and mortality in Hospital acquired infection. 


\section{Acknowledgement}

We owe our great thanks to DBT, Tezpur for financial support in our study.

\section{References}

Bergogne-Berezin, E., Towner, K.J. 1996. Acinetobacter spp. As nosocomial pathogens: microbiological, clinical and epidemiological features. Clin. Microbiol. Rev., 9: 148-65.

Biglari, S., Hanafiah, A., Ramli, R., Rahman, M.M., Khaithir, T.M.N. 2013. Clinico-epidemiological nature and antibiotic susceptibility profile of Acinetobacter species. Pak. J. Med. Sci., 29(2): 469-473.

Chakraborty, B., Banerjee, D., Chakraborty, B. 2011. Acinetobacter baumannii: no more a choosy intruder. Indian J. Med. Sci., 65(8): 344-348.

David, L., Paterson, Samuel, I., Miller. 2012. Chapter 150, In: Harrison's Principles of Internal Medicine, $18^{\text {th }}$ Edition, (Mc Graw Hill) Editors: Dan longo, Dennis Kasper, J. Larry Jameson, Anthony Fauci, Stephen Hauser, Joseph Loscalzo, Vol.1, pg 1258-1260.

Dima, S., Kritsotakis, E.I., Roumbelaki, M., et al. 2007. Device-associated nosocomial infection rates in intensive care units in Greece. Infect. Control Hosp. Epidemiol., 28: 602-5.

Fournier, P.E., Richet, H. 2006. The epidemiology and controlof Acinetobacter baumannii in health care facilities. Clin Infect. Dis., 42: 692-9.

Lolans, K., Rice, T.W., Munoz-Price, L.S., Quinn, J.P. 2006. Multicity outbreak of carbapenem-resistant Acinetobacter baumannii isolates producing the Carbapenemase OXA40. Antimicrob. Agents Chemother., 50: 2941-5.
Muktikesh Dash, Sanghamitra Padhi, Swetlana Pattnaik, Indrani Mohanty, and Pooja Misra. 2013. Frequency, risk factors, and antibiogram of Acinetobacter species isolated from various clinical samples in a tertiary care hospital in Odisha, India vicenna J. Med., 3(4): 97-102. doi: 10.4103/2231-0770.120501

Munoz-Price, L.S., Robert, A., Weinstein. Acinetobacter Infection Current concepts. N. Engl. J. Med., 358: 12711281.

Muntasir, I., Omer, Samia, A., Gumaa, Abdullatif, A., Hassan, Khaled, H., Idris, Osama, A., Ali, Mustafa, M., Osman, Mahmmoud, S., Saleh, Nagla, A., Mohamed, Mustafa, M., Khaled. 2015. Prevalence and Resistance Profile of Acinetobacter baumannii Clinical Isolates from a Private Hospital in Khartoum, Sudan. American $J$. Microbiol. Res., vol.3, No 2, 76-79.

Namita Jaggi, Pushpa Sissodia, Lalit Sharma. 2012. Acinetobacter baumannii isolates in a tertiary care hospital: Antimicrobial resistance and clinical significance, J. Microbiol. Infect. Dis., 2(2): 57-63.

Patwardhan, R.B., P.K. Dhakephalkar, K.B. Niphadkar, B.A. Chopade. 2008. A study on nosocomial pathogens in ICU with special reference to multiresistant Acinetobacter baumannii harbouring multiple plasmids, Indian J. Med. Res., pp 178-187.

Poonam Sharma, Yousuf Ul Ashir, Sarjiwan Kaur, Parmeet Kaur, Aruna Aggarwal. 2015. Emerging antimicrobial resistance and clinical relevance of Acinetobacter isolates in a tertiary care hospital of rural area of Punjab, India. J. Microbiol. Antimicrobial. Agents, 1(1): 8-12. 
Prasantha, K., S. Badrinath. 2004. Invitro susceptibility susceptibility pattern of Acinetobacter species to commonly used cephalosporins, quinolones and aminoglycosides; Indian J. Med. Microbiol., 22(2): 97-103.

Rahul Kamble, et al. 2012. Acinetobacter species in Health Care setting: Clinical significance and Antimicrobial sensitivity Int. J. Curr. Microbiol. App. Sci., 4(4): 861-869.

Schreckenberger, P.C., Daneshvar, M.I., Weyant, R.S., Hollis, D.G. Acinetobacter, Achromobacter, 2007. Chryseobacterium, Moraxella, and other non fermentative gram negative rods. In: Murray PR, Baron EJ, Jorgensen JH, Landry ML, Pfaller MA, eds. Manual of clinical microbiology. 9th ed. Washington, DC: ASM Press, 770802.

Sinha, N., J. Agarwal, S. Srivastava, M. Singh. 2013. Analysis of carbapenem-resistant Acinetobacter from a tertiary care setting in North India; Indian $J$. Med. Microbiol., 31(1): 60-63.

Sivaranjani, V., Umadevi, S., Srirangaraj, S., Kali, A., Seetha, K.S. 2013. Multidrug resistant Acinetobacter species from various clinical samples in a tertiary care hospital from South India. Australasian Med. J., 6(12): 697-700.

Zakuan Zainy Deris, Mohd Nazri Shafei, Azian Harun. 2011. Risk factors and outcomes of imipenem resistant Acinetobacter bloodstream infection in North-eastern Malaysia. Asian Pacific J. Trop. Biomed., 1(4): 313-5.

\section{How to cite this article:}

Harekrishna Nath and Dipa Barkataki. 2016. Study of Acinetobacter Isolates from Clinical Specimens in Tertiary Care Hospital and their Antimicrobial Susceptibility Pattern. Int.J.Curr.Microbiol.App.Sci. 5(10): 842-848. doi: http://dx.doi.org/10.20546/ijcmas.2016.510.092 